\title{
Research on the Error Detection Technology of Complex Parts based on Improved Bee Colony Algorithm
}

\author{
Jianghua Ge, Huihui Zhang, Yaping Wang, Xiulin Sui, Yongguo Sun \\ Machinery and Power Engineering College \\ Harbin University of Science and Technology, Harbin, China \\ gejianghua@sina.com
}

\begin{abstract}
For Parts of complex structures with multiple characteristics, traditional methods are often difficult and inefficient, so the three-dimensional detection techniques have been developed. But the detection used for the complex structural components still get some problems, such as : it's difficult to collect data of complex structural components in the data collection process, because of size is always limited; features of parts retained difficultly in data processing; there are many iterations and calculations in the optimization algorithm of geometric error evaluation, and the search direction is blind. In this paper, the improved colony algorithm is used to get the cylindrical error by establishing the mathematical model with the point cloud data that has been processed. First, collect data acquisition of more structural parts with hand-held laser scanners; Secondly, denoise and streamline the raw point cloud collected before, and then make registration and alignment with the actual CAD model of part; Finally, according to the resultant point cloud data above, in accordance with the principle of minimum condition assessment, establish evaluation model of cylinder error based on improved bee colony algorithm. Experimental results show that for various structural parts, the precision of this detection method is high enough, and detecting speed is also fast.
\end{abstract}

Keywords: complex structural components; three-dimension testing; point cloud data; improved bee colony algorithm; mathematical model

\section{Introduction}

Aerospace industrial development is very rapid in our country, the designs of some parts in the field of aircraft, automobile become more and more complex, therefore the requirements of complex parts detection accuracy are also getting higher and higher [1]. In the traditional methods, error of manual measurement method with measuring tool is big and efficiency is also low, in the case manual measurement method has been unable to meet the standards of modern industrial measurement, the three-dimensional detection technology has began to be developed.

Xiaojuan Sun [2] in Harbin University of science and technology detected the contour surfaces and error of gear by three-dimensional coordinate measuring machine, Xiaoli Liu and Xiang Peng et al.[3] in Shenzhen University who use three-node optical sensor to establish a data grid to obtain some features size information of shell in large size, match with computer-aided design (CAD) model through iterative closet point algorithm, and then get the distance between CAD model and the point of measurement, so that the pseudo-color pictures of model error distributions can be drawn according to the distance. Jinlong Shi and Qiang Qian et al. [4] scan different parts of the steel sheet by using three-dimensional optical 
scanner, and then spliced part of the data, this test method is applied to the outer plate processing vessel. Yuling Cao and Cunzhu Chen et al[5] proposed the three-dimensional detection method of industrial CT scanning, multiple continuous two-dimensional image can be gotten by using industrial CT to scan the casting, then the three-dimensional image can be reconstructed, and finally a three-dimensional model of the casting can be gotten, the internal defects of casting can be determined according to the three-dimensional image model to achieve the rapid non-destructive testing. Guangping Ta and Shijie Wang[6] proposed the detection method of oil tank structure based on three-dimensional laser scanner, which used non-contact measurement, by analysing and processing point cloud data from threedimensional laser scanner to make the accuracy analysis of structure, this detection method is efficient, meanwhile it can meet the requirements of detection accuracy and solve the detection problem of large parts effectively. Wuhan University and Wuhan Supresoft Software Inc. have developed a three-dimensional detection measurement system easy and high-precision--VPSS Inspection System vision inspection system[7]. Aihong Gong and Xueliang Zhou[8] make the engine crankshaft as the main research object, combined with the measurement software ARCO CAD from three-dimensional coordinate measuring machine to test mathematical model of crankshaft, analysis the information of crankshaft's mathematical model and three coordinate measuring technology and extraction methods of the theoretical value. In summary, in the three-dimensional detection technique, most of the domestic researchers focus more to the point cloud data and the alignment of parts model, and the pseudo-color pictures of error distribution obtained by reverse software, not give error analysis of form and position directly. However, in the quality inspection process of complex structural components not only requires 3D error comparison technology, but also need extraction techniques of form and position errors about complex structural components measured.

In cylindricity error of parts on the establishment of mathematical model and algorithm research, scholars from the United Kingdom Dgchetwyhd[9] established cylinder evaluation method, the main evaluation method has a minimum circumcircle, maximum inscribed circle method and minimum zone method. TsRmurthy et al[10] on the establishment of part cylindrical error of model, used the least squares method, orthogonal polynomial method, least squares method vertical surface expansion methods and so on as evaluation methods of measurement, these types of methods were used to assess the test, different data of test were compared finally, the smallest error value of cylinder is perpendicular evaluation method of least squares method. Japanese scholar[11] evaluated the roundness and cylindrical errors with the least square method and minimum zone method, selected two methods randomly to analyze and compare the cylindricity error data measured, concluded that final result of the two error evaluation was very diffrent. American scholar u.Roy[12] proposed cylindricity error evaluation method of the minimum zone base on computer geometry, according to the measurement obtained properties of the plane point cloud data set Vorohoi graph and determined the exact location of the minimum area circle center directly, concluded that accuracy of cylindrical error values were much higher. American scholar KISten. Carr et al[13] established variety of cylindrical error evaluation model, mainly involving mathematical calculation method included a minimum area, minimum and maximum circumcircle law inscribed circle method, under the principle of linear programming approach, for the accuracy of these error evaluation model, concluded that the error precision of cylindrical error evaluation based on the three mathematical methods was very high, and required the standard of production and processing. In summary, for multi-feature points, face complex structural components product, error evaluation algorithm of form and position based on measurement of point cloud data still exists problems, such as calculation results are 
mostly approximations, algorithm iterations are too many, it's easy to fall into local optimal solution, and algorithm computation is complex and so on.

\section{Acquisition of Complex Structure Parts Point Cloud Data}

According to the characteristics of complex parts, use hand-held scanning instrument of laser triangulation measure principle to acquire point cloud data of complex parts, Figure 1 is point cloud data acquisition of complex parts with hand-held laser scanner.

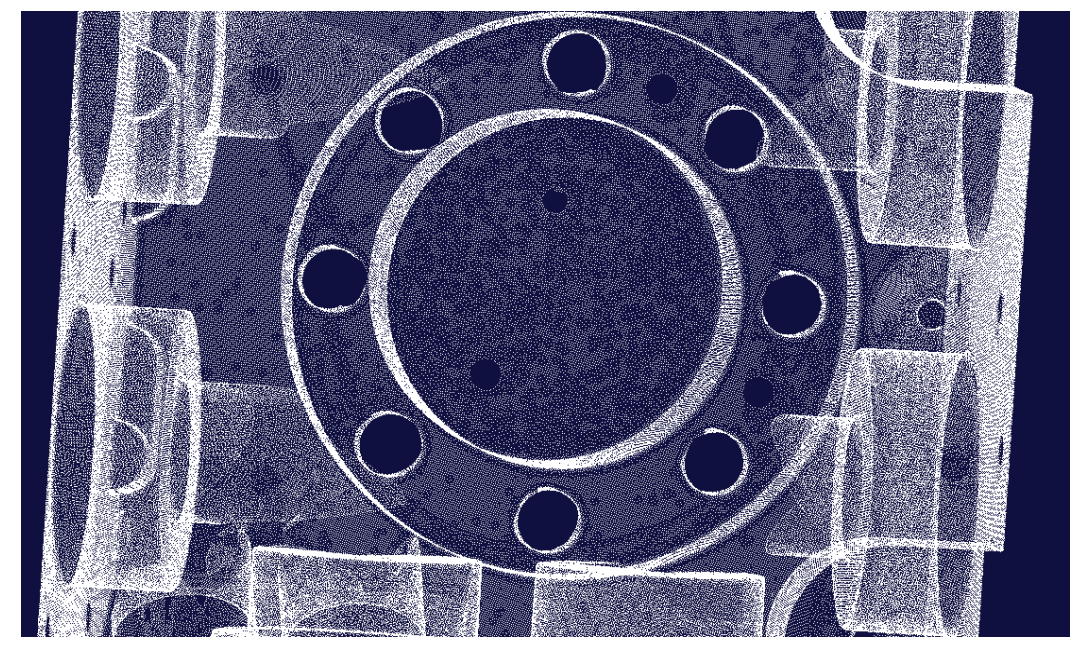

Figure 1. Point Cloud Data Acquisition of Complex Parts

\section{Cloud Data Process of Complex Parts}

\subsection{Noise point process}

Large point cloud data can be obtained by 3D laser scanning, there must be a part of noise point in this large data, that is, unnecessary interference data. Therefore, in order to improve the accuracy of subsequent data processing, we must first carry out the noise reduction. The point cloud data acquired by the 3D laser scanner is usually processed by the filtering algorithm. In data processing, the simplest noise processing method is using human-computer interaction, this method can prevent the filtering algorithm to interfere the original point cloud data on the accuracy, the image of the point cloud data can be directly displayed on the computer display, the operator is able to judge noise point position clearly and eliminate the noise point, this method of noise elimination is more intuitive. This paper use of the software to directly display the measured point cloud data, then carry on the real-time observation and processing of point cloud data image, purple selected point in Figure 2 is the point cloud data that will be handled as the noise point. 


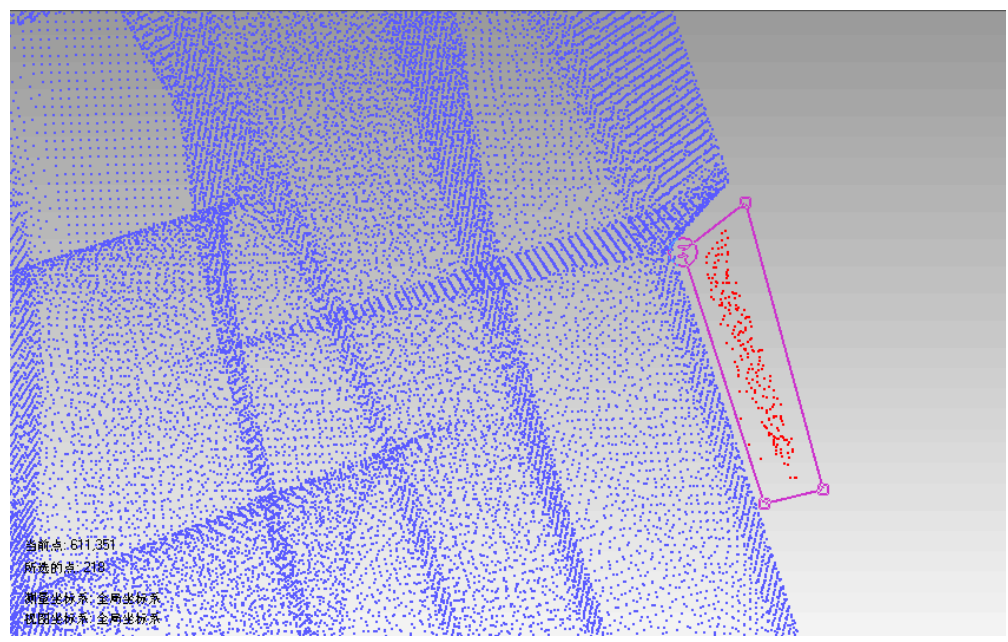

Figure 2. Process of Noise point

\subsection{Hybrid Simplification Based on Curvature Reduction}

3D laser scanner can scan tens of thousands of coordinate data point set in unit area onetime, if scans for large parts of the complete, a rallying point quantity may reach to millions. So after scanning, the acquisition point cloud data will be simplified, because the amount of point cloud data over large will lead to the decline of the operational speed of the computer, that can also cause surface smooth degree to decline. So the original point cloud data should be streamlined, that is, under the premise that ensure the characteristics of the cloud data model, streamline the unnecessary redundant points.

Now, there is no streamlined algorithm can also meet the sampling precision, accuracy and speed for the point cloud data of multi characteristics parts, so the current algorithms are all unsatisfactory. In this chapter, use the mixed sampling method combined with random sampling and curvature sampling, this method is good comprehensively. It not only solved the shortcoming that random algorithm in randomization cannot control accuracy and can not guarantee the surface characteristics, and also overcame the defects that the curvature simplification will be removed in a large number of points set, resulting in losing some characteristics of point cloud data and generating voids easily, the simplification method can not only make the advantages of both possibly fuse but also weaken the disadvantages of both at the greatest extent proved to be feasible in practical application.

The implementation steps of the algorithm are as follows:

Step1: read the point cloud into the software, set the sampling percent to the specified by theuser.

Step2: determine whether the percentage is less than the set value, such as the set value is $10 \%$. If it's less than the set value, then only carry on the random sampling of percentage and jump directly to step 6; if it is higher than the set value, then carry on random sampling of set value and then execute the next step.

Step3: after random sampling of point cloud, carry on its rest specified percentage of curvature sampling (set value).

Step4: some points have been done to remove the tag, sample these points are randomly, take part of their small, remove and delete tags.

Step 5: remove the point which has the removed tag from the point cloud.

Step 6: output the point cloud which has been sampled, end. 
A comparative analysis is made on the basis of the simple random, curvature and simplification of curvature. The comparison and analysis are shown in Figure 3.

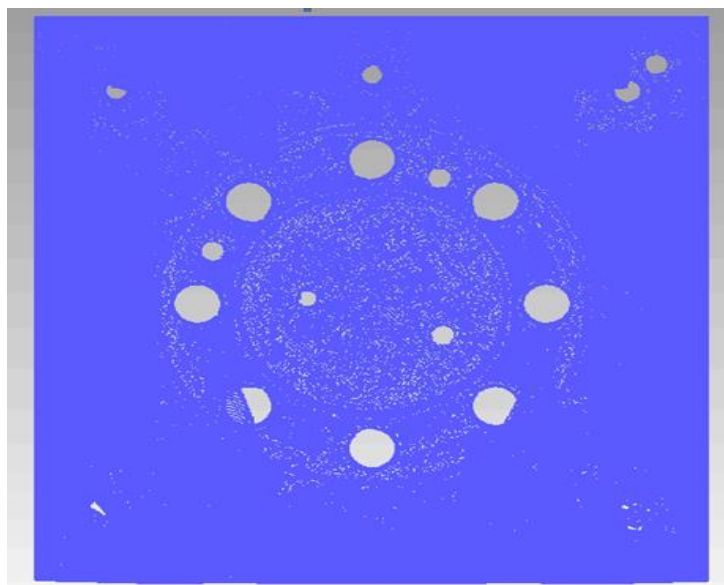

a) Original point cloud data

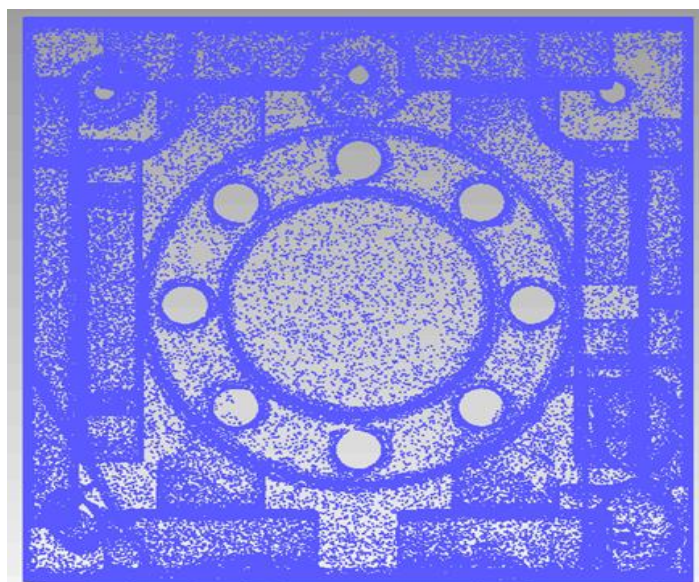

b) Mixed reduction effect

Figure 3. A hybrid Reduction based on Curvature Reduction

Figure 3 shows that the hybrid compact not only retains most of the point cloud features, and there is no gap. On the sampling rate, the speed of the latter two is far more slowly than the random sampling, but relatively speaking, the mixed sampling has more advantages than the curvature sampling.

\subsection{Compound Alignment based on Feature Alignment}

The point cloud data processed should be aligned with CAD model, after alignment the error between the physical parts and design model can be obtained by corresponding technology, then the error of complex parts were detected.

The research object of this paper is multi feature of complex parts, parts are generally not only has hole, cylinder, cone and plane feature, some complex parts also has the feature of complex free surface, and alignment method commonly used now cannot be used for complex curved surface and the basic features of accurate alignment at the same time. Therefore, this section presents a composite method based on feature alignment and the best fitting method of alignment together. The composite alignment method is a-coarse-registration based on feature alignment for the point cloud data and the model, and then uses the best fitting alignment to carry on the accurate registration. The effect of registration is shown in Figure 4. 


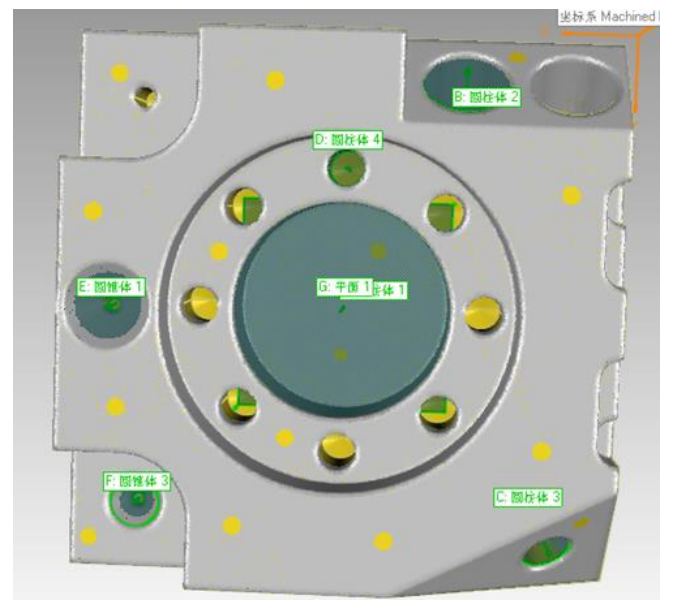

a) Feature alignment

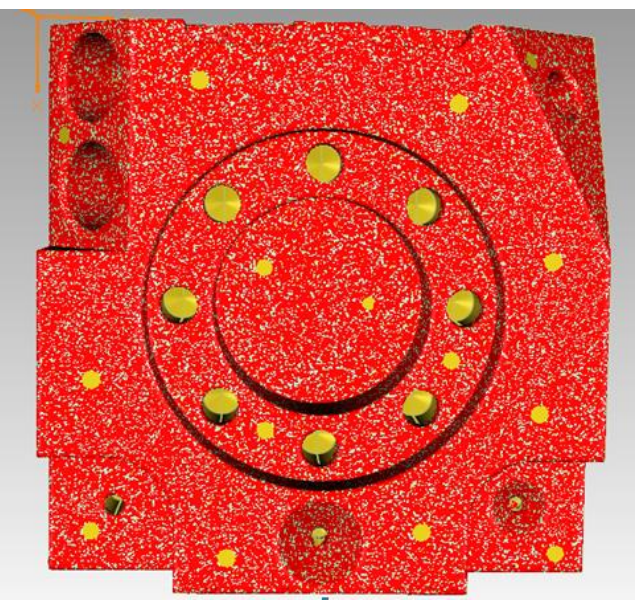

b) Best fit alignment

Figure 4. Composite Alignment based on the Alignment Technology of Characteristics

\section{Error Evaluation of Complex Parts}

\subsection{D Error Comparisons of Complex Parts}

Post the alignment model into geomagic qualify, through the comparison of the 3D part of the overall situation can be gotten, a comprehensive, clear and intuitive, image of chromatographic results figure is gotten, as shown in Figure 5.

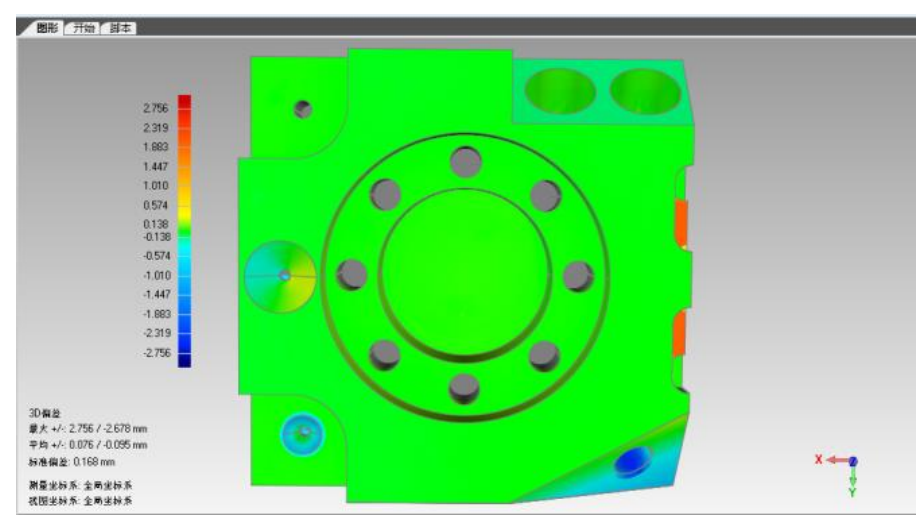

Figure 5. 3D Comparison of Color Error Analysis

This is only color error diagram, in order to see some of local deviation more clearly, we need to create a comment on some points of the detailed analysis, the analysis results are shown in Figure 6. 


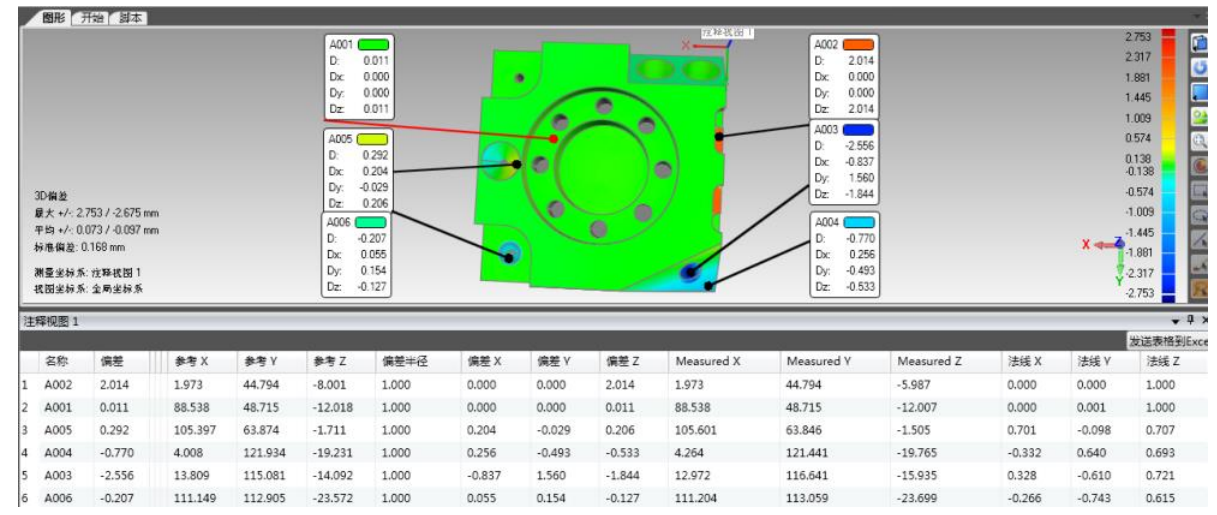

Figure 6. Sample Point Analysis of Detection Report

Because of the error range of setting is $4 \mathrm{~mm}$, from the comparison of the results, it can be seen that the maximum deviation above is $2.735 \mathrm{~mm}$. The maximum deviation below is $2.675 \mathrm{~mm}$, average deviation above is $0.073 \mathrm{~mm}$, average deviation below is under $-0.097 \mathrm{~mm}$.

\subsection{Error Evaluation of the Circular Cylinder based on the improved bee Colony Algorithm}

1) Mathematical model of circular cylinder error evaluation

Cylindrical error is usually meaned the amount of change by the actual the cylindrical surface and ideal cylindrical surface. Therefore, the first condition of cylindrical error evaluation is to find the position of the ideal cylindrical surface, the confirmation of ideal cylindrical surface should be based on the principle of minimum condition, that is to say, the largest amount of variationthe between measured actual elements and ideal elements is a minimum.

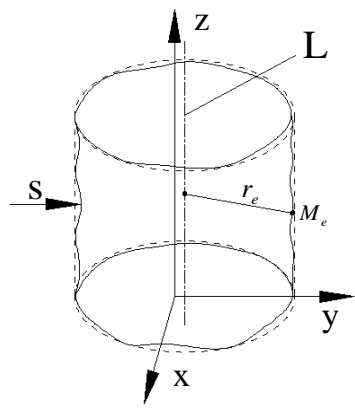

\section{Figure 7. Error Model of Cylindrical Degree}

The space coordinates in Figure 7, assume that ideal cylindrical axis is L, it's location relationship is indicated by two parameters a,b, it's direction relation is indicated by two parameters $\mathrm{p}, \mathrm{q}$, then the linear equations formula of this axis $\mathrm{L}$ is showed in(1).

$$
\frac{x-a}{p}=\frac{y-b}{q}=\frac{z}{1}
$$

Coordinate $M_{e}\left(x_{e}, y_{e}, z_{e}\right), e=1,2, \cdots, n$ is a point of the actual cylinder $\mathrm{S}, \mathrm{n}$ is the amount of points, and the distance between the points $M_{e}$ and the axis $L$ is shown in the formula (2). 


$$
r_{e}=\frac{\left\|\begin{array}{ccc}
i & j & k \\
x_{e}-a & y_{e}-b & z_{e} \\
p & q & 1
\end{array}\right\|}{\sqrt{p^{2}+q^{2}+1}}
$$

When evaluate the error of the cylinder with the minimum area rule, the actual cylindrical surface $\mathrm{S}$ is coated with two ideal cylindrical surfaces. If the radius difference of the two cylindrical surfaces above is minimum, the area included by these two cylindrical surfaces can be said to be the minimum area $Z_{e}$. At this time, the radius difference $t$ between the two cylindrical surfaces is the error of the cylindrical degree that is actually measured. According to the provisions of the minimum area principle, meanwhile substitute the formula (2), then the optimal objective function $f(a, b, p, q)$ is shown in (3).

$$
\begin{aligned}
& f(a, b, p, q)=\max r_{e}-\min r_{i}= \\
& \max \frac{\sqrt{\left(y_{e}-b-q z_{e}\right)^{2}+\left(x_{e}-a-p z_{e}\right)^{2}+\left[\left(x_{e}-a\right) q-\left(y_{e}-b\right) p\right]^{2}}}{p^{2}+q^{2}+1}- \\
& \min \frac{\sqrt{\left(y_{i}-b-q z_{i}\right)^{2}+\left(x_{i}-a-p z_{i}\right)^{2}+\left[\left(x_{i}-a\right) q-\left(y_{i}-b\right) p\right]^{2}}}{p^{2}+q^{2}+1}
\end{aligned}
$$

As shown above, $e, i=1,2, \cdots, n, n$ is a measure of the number of coordinates.

According to the meaning of the cylindrical error, the formula (3) is an objective optimization function of cylindrical error that satisfy the provisions of the minimum zone. So, the problem of cylindrical error can be changed to the optimization of parameters $a, b, p, q$, so that the target optimization function $f(a, b, p, q)$ can obtain the minimum value.

\section{2) Colony algorithm of tabu search strategy}

Bee colony algorithm is proposed by a Turkish scientists Karaboga in 2005, through observing the bees search in honey process he got the optimization algorithms for the best nectar, because the algorithm in many research fields have gained performance than the other algorithms better, so artificial bee colony algorithm caused the attention of researchers. In the traditional bee colony algorithm, it is usually divided into 3 kinds of bees: employment, observation and reconnaissance.

For the traditional artificial bee colony algorithm, in the last stage of iteration calculation, the employed bees will search the nectar have given up in the past, thereby increase the post calculation workload, affect the speed of search for the optimal solution, so with tabu strategy evaluate the cylindrical error and make sure that when the employed bees in the progress of later search optimal solution, bees would not search the local optimal solution has given up again. Tabu strategies means that with the form of tabu list, the local optimal solution have given up is stored, at the same time, mark them so that you can avoid to search these local optimal solution late again. After adding tabu strategy, when bee colony algorithm update the nectar, if there is a higher degree of nectar nearby, the employed bees will choose the higher degree of nectar to update, and will also detect the new nectar to see whether the new nectar is in the tabu list, if it's not in the tabu list, then develop the incremental computation of revenue, otherwise it will be put into update of the tabu list. 
The error of the cylindrical degree based on the improved bee colony algorithm is as follows:

Step1: First, initialize the number of swarm, the employed bees and the observed bees are equal to the number of the nectar, each accounted for 40, locate 500 iterations.

Step2: Unify the four parameters $a, b, p, q$ of mathematical model of the cylindrical error, to make the computer calculation convenient, shown in the following formula (4).

$$
\left\{\begin{array}{l}
a=\min \left\{X_{i}\right\}+u\left(\max \left\{X_{i}\right\}-\min \left\{X_{i}\right\}\right) \\
b=\min \left\{Y_{i}\right\}+u\left(\max \left\{Y_{i}\right\}-\min \left\{Y_{i}\right\}\right) \\
p=-1+2 u \\
q=-1+2 u
\end{array}\right.
$$

The $u$ in the formula is the random number from 0 to $1, X_{i} 、 Y_{i}$ is the coordinates of the measured point data.

Step3: Put the employed bees into a nectar randomly, solve the benefit of this nectar. The calculation of benefit shown in the following formula (5).

$$
\text { fit }_{i}=\frac{1}{1+f(i)}
$$

Step4: In each iteration of The bee colony algorithm, according to the benefit of nectar, observed bees choose the nectar through roulette way, the probabilities of choice are given in the formulate (6) .

$$
P_{i}=\frac{f i t_{i}}{\sum_{n=1}^{S N} f i t_{n}}
$$

Step5: Through the formula (7) update the nectar, meanwhile according to the benefit of nectar, determine whether choose the new nectar.

$$
\begin{gathered}
\text { newX }=X_{i j}+\partial \times\left(\mathrm{X}_{\mathrm{ij}}-\mathrm{X}_{\mathrm{ik}}\right), \mathrm{j} \neq \mathrm{k} \\
\left\{\begin{array}{l}
X_{\mathrm{ij}}=n e w X, \quad \text { newfit }>\text { fit } ; \\
X_{i j}=X_{i j}, \quad \text { others }
\end{array}\right.
\end{gathered}
$$

$\left\{\begin{array}{lc}\text { DetectFitInc } & \text { newfit } \notin G ; \\ \text { TabuState } & \text { else }\end{array}\right.$

In the type above ${ }^{\partial \in(0,1)}, \mathrm{G}$ indicate the tabu list, DetectFitInc indicate benefit change detection of new nectar, TabuState indicate that record the nectar on the update to the tabu list.

Step 6: If the employed bees iterate limit in a nectar, and still can not find the nectar benefit improve significantly, then the employed bees will give up the nectar, turn into the observed bee, at the same time put the local optimal solution into the tabu list.

Step7: Check if the algorithm require the condition of automatic stop, if it is, then get the cylindrical error optimal solution of the entity model, if not, then go to step 3 iterate arithmetic again, until meet the termination conditions. 
3) Calculation of cylindrical error evaluation

In order to assess cylindrical error of the complex parts, this paper selected a hole to measure randomly, it's coordinates of the point cloud data is shown in Table 1.

Table 1. Data of the Measurement

\begin{tabular}{c|c|c|c}
\hline No. & X & Y & Z \\
\hline 1 & 24.755 & 49.419 & -5.079 \\
\hline 2 & 26.964 & 44.248 & -3.523 \\
\hline 3 & 23.520 & 53.478 & -5.401 \\
\hline 4 & 22.841 & 57.59 & -5.824 \\
\hline 5 & 22.599 & 60.408 & -5.613 \\
\hline 6 & 22.638 & 65.118 & -6.896 \\
\hline 7 & 24.183 & 73.871 & -7.629 \\
\hline 8 & 28.294 & 83.240 & -10.289 \\
\hline 9 & 31.129 & 87.278 & -3.914 \\
\hline 10 & 32.711 & 89.191 & -6.324 \\
\hline
\end{tabular}

Large amount of simulation computation has been done to the cylindrical error of the complex parts with the improved artificial colony algorithm, first make the initial swarm size be 100 , lead bees and follow bees are both 50 , and define the food source for 50 , the number of iterations is 300 , set Limit to 40 times, run 50 times independently. With the improved artificial colony algorithm, get the cylindrical error $0.114 \mathrm{~mm}$, the average operation time is $1.4 \mathrm{~s}$, with the cylindrical measuring instrument to test the characteristics of the hole, can get that cylindrical error is $0.08298 \mathrm{~mm}$, the difference of cylindrical error with algorithm calculates is $0.03 \mathrm{~mm}$, can meet the requirement.

\section{Conclusion}

This paper researched on the point cloud data acquisition and processing technology of parts with characteristics, mixture of streamline based on the curvature streamline and composite alignment technique based on the characteristics of the alignment are proposed according to the characteristics of complex parts, compared and analysed the 3D error through the software to point cloud data has been processed, finally proposed the colony algorithm based on the strategy of taboo to evaluate of the cylindrical error, the experimental results show that not only the evaluation accuracy meet enterprise actual requirement, but also it's much faster than the other testing method, therefore it has got the important significance in the process of the enterprise production.

\section{Acknowledgements}

University Science Park Entrepreneurial Talent of Innovation Talents of Science and Technology of Harbin Application Technology Research and Development Project (2013RFDXJ001)

\section{References}

[1] B. Liu. Surface 3d micro profile measuring technology research based on light bulb phase-shift interfering method D.HUST, (2013).

[2] X. Sun. 3d coordinate measuring instrument detection of Gear's parameters and precision D.HUST, (2006). 
[3] X. Liu, X. Peng, Y. Yin. 3d optical automatic detection on the surface of large size shell J. Acta Optica Sinica, (2011), vol. 31, no. 3, 0312006-1-0312006-7.

[4] J. Shi, Q. Qian, L. Pang. 3d measurement and stitching of large Scale J. Optics and Precision Engineering, (2014)(05) : 1165-1170.

[5] Y. Cao, Cunzhu Chen. Industrial CT three-dimensional detection technology of casting J. Computed tomography (CT) theory and application research, (2003)(8) : 36-39.

[6] G. Ta, Shijie Wang. The ground three-dimensional laser scanning technology in the application of storage tanks J. Mine surveying, (2014)(06) : 60-62.

[7] H. Guan. Three dimensional error analysis of testing system based on industrial rice gold pieces of sequence images D. WuHan: Wuhan university, (2005).

[8] A. Gong, Xueliang Zhou. Three-dimensional detection based on mathematical model of the engine crankshaft forgings J. Equipment Maintenance Technology, (2007)(3): 24-27.

[9] D G Chetwynd . Application of linear programming to engineering metrology J. Proc Instn Mech Eng, (1985), 199(BZ), pp 93-100.

[10] Murthy T S R, Rao SY. A simple approach for evaluation of cylindrical surface J. Annals of the CIRp, (1981), 30(1):441-444.

[11] T. Tsukada, kanada T, Okuda K. An evaluation of roundness from minimum zone center by means of an optimization techniques J. Bull Japan Soc of Prec Eng, (1984), vol. 18, no. 4, pp. 317-322.

[12] U. Roy, X. Zhang. Establishment of a pair of concentric circles with the minimum radial Separation for assessing roundness error J. Computer-aided Design , (1992), vol. 24, no. 3, pp. 161-168.

[13] K. Carr, P. Ferreira. Verification of form tolerances Part 2 : Basic issues Cylindricity and Straightness of a median line J. Preeision Engineering (1995), vol. 17, no. 2, pp. 44-55. 
International Journal of Security and Its Applications Vol.9, No.12 (2015) 